\title{
Theory of pleiotropic action of biologically active compounds and medicines-Basic principles and practical application
}

\author{
N. A. Bizunok \\ Pharmacology Department, Belarusian State Medical University, Minsk, Republic of Belarus \\ Email: bizunokna@yandex.ru \\ Received 27 May 2013; revised 27 June 2013; accepted 5 July 2013 \\ Copyright (C) 2013 N. A. Bizunok. This is an open access article distributed under the Creative Commons Attribution License, which \\ permits unrestricted use, distribution, and reproduction in any medium, provided the original work is properly cited.
}

\begin{abstract}
This article represents the main positions of the theory of pleiotropic action of biologically active compounds (BACs) and medicines, which has been designed by the author based on her own experimental researches. The term "pleiotropy" means the ability of the BACs and medicines to implement more than one mechanism of action resulting in the specific biological (pharmacological) effect. The interaction of these mechanisms forms a distinct pattern of biological response (pleiotropic pattern), which reflects the change in his character with the increased dose (concentration)-dependent efficacy of BACs and medicines. The article consists of description of different pleiotropic patterns established in experiments on the model of reactive oxygen species (ROS) generation by macrophages dependent on activity of specialized enzyme called Nox2-NAD(P)H oxidase (Nox2, EC 1.6.3.1). Moreover, it consists of explanation of pharmacodynamic nature of pleiotropic patterns by means of application Chou-Talalay median effect equalization and combination index (CI) theory. The novel theory explains unsolved until now universal aspects of activity BACs and medicines, such as slope angles of "doseeffect" dependences in the conditions relevant in vivo, and it is of fundamental interest. However, it has applications in experimental pharmacology, as it allows defining the choice of the individual compounds and combinations, modulating the trust effect selectively and efficiently. This knowledge opens up new approaches to medicines discovery and evaluation, their rational dosing and combining.
\end{abstract}

Keywords: Reactive Oxygen Species (ROS); Nox2-NAD(P)H Oxidase (Nox2); Slope Angles of Dose-Effect Dependences; Pleiotropy; Pleiotropic
Patterns; Polytropism; Pharmacological Affect Nature; Medicines Combinations; Novel Theory of Biologically Active Compounds (BACs) and Medicines Action

\section{INTRODUCTION}

Rapid development of molecular pharmacology and organic chemistry in the XX century has given mankind the explosive growth of the absolute number of new medicines, but, as the result of clinical studies, many prototypes, emerged at the dawn of experimental pharmacology, proved to be more effective than those that have seemingly improved pharmacodynamic and pharmacokinetic profile. Since efficiency is the main criterion of the clinical medicine value, such drugs have become the standard (exemplar). Our research demonstrates that a high therapeutic efficacy of standard treatment may be due to the special nature of their biological effect, based on pleiotropic action.

The term "pleiotropy" borrowed by the author from the terminology of genetics, in the best possible way reflects the nature of the biologically active compounds (BACs) and medicines in complex biological systems and in vivo, organized by certain biological (pharmacological) effect induction. In genetics pleiotropy refers to the impact of a single gene in the development of several phenotypic traits. In this context, pleiotropy means the ability of the BACs and medicines to implement more than one mechanism of action resulting in the specific biological (pharmacological) effect. The interaction of these mechanisms forms a distinct pattern of biological response (pleiotropic pattern), which reflects the change in his character with the increased dose (concentration)-dependent efficacy of BACs and medicines. Further in this paper, pleiotropy will be discussed in relation to antioxidant compounds for the sole reason that the model used for its study, was the cells, carrying enzyme 
Nox2-associated NAD(P)H-oxidase (Nox2, EC 1.6.3.1) responsible for reactive oxygen species (ROS) generation within them, the search of approaches to which became the original paradigm of the research. However, the patterns revealed by this pleiotropic activity have the universal biological value and can be applied in any relevant conditions. Nevertheless, antioxidants is a group of drugs most critical for understanding of the pleiotropic activity nature, as clarification of this issue helps to avoid many mistakes and disappointments in their clinical application.

The fundamental preconditions of the theory of BACs and medicines pleiotropic action lie in the nature of "dose (concentration)-effect" dependence shown in Figure 1 for normal (a), semi-log (b) and probit coordinates (c).

The latter two measurements are traditionally used in experimental pharmacology to analyze the quantitative parameters of the medicines. The main ones are the maximum effect $\left(\mathrm{E}_{\max }\right)$, effective doses (concentrations) $\mathrm{ED}_{16}-\mathrm{ED}_{84}\left(\mathrm{EC}_{16}-\mathrm{EC}_{84}\right)$, allowing to estimate the activity of the effector and the slope coefficient of the "dose (concentration)-effect" (m) dependence [1,2]. Despite the fact that the slope angle of "dose (concentration)-effect" dependence is taken into account in the evaluation of pharmacodynamic interactions, its determinants are not known and the reasons for the variability still require adequate explanation. Attempts to make the analysis of that kind were taken in the first half of the twentieth century by Archibald Hill, a British physiologist, who offered to consider them as a result of interreceptor interactions within the frames of the receptor theory, developed intensively at that time, and hypothesized about conjectured receptor cooperativity. He also introduced a convenient coordinate system to analyze the slope angles of "dose (concentration)-effect" (a variant of probit analysis). Since then, the idea of receptor cooperativity was widely used in biochemistry and molecular pharmacology. However, the accumulation of the contradictory results in the experiments with receptor agonists gradually led to doubt that receptor cooperativity is the only explanation of differences in the slopes of the dose dependence, especially since it does not include compounds realizing non-receptor activity mechanisms, also inherent in these differences. Nevertheless, the extrapolation to receptorology of enzymological analytical approaches has led to the fact that for the quantitative analysis of ligand-receptor interactions Lainuiver-Berk and Scatchardt conversions become widely used thus allowing determine $\mathrm{Kd}$ (dissociation constant) for many BACs and medicines based on the slop analysis of the transformed "dose-effect" dependence. Soon, it was revealed that the "true" $\mathrm{Kd}$ calculated in biochemical experiments, and "apparent" Kd calculated in pharmacological experiments can vary by several orders of magnitude $[3, \mathrm{pp}$. 14-15]. This controversy led to the idea of free receptors and attempts to modify the classical theory. Among the most significant of these efforts the theory by R.B. Stephenson (1956), was the most recognized and greatly

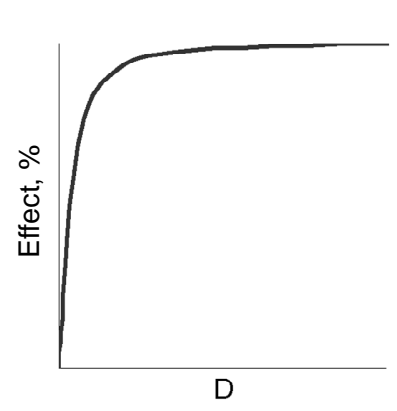

(a)

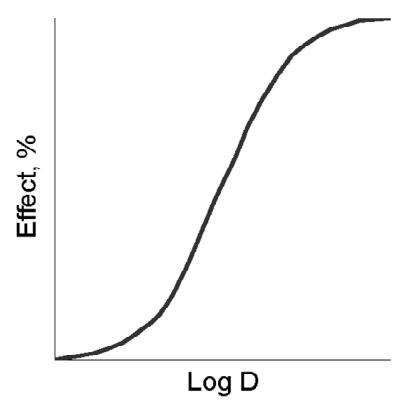

(b)

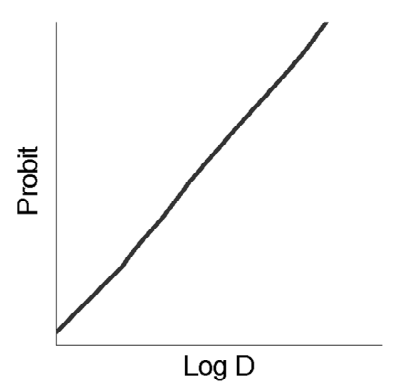

(c)

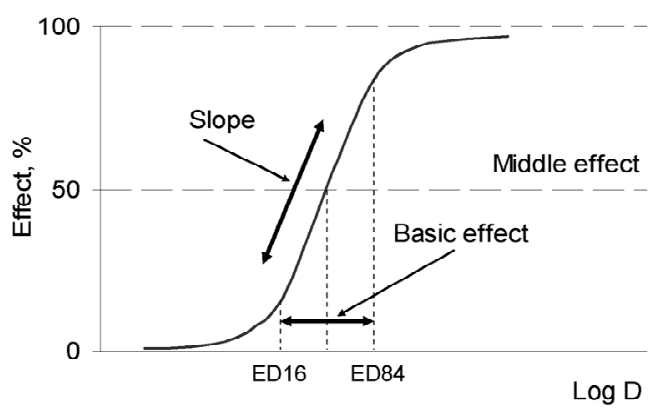

(d)

Figure 1. The "dose-effect" dependence in the normal (a), semi-log (b) and probit (c) coordinates, the main quantitative parameters of the above dependence $(d)$. 
complemented to the classical theory. It was R.B. Stephenson who first proposed the idea that the connection between the stimulus and the effect is a property of tissue, and not the medicine [4], thus, the effect of medicines was placed outside the receptor theory. Significant contribution to the development of receptorology was also made by W.D. Paton (1961) [5], A.T. Karlin (1967) [6], D Colquhoun $(1973,1998)$ [7,8], A. DeLean et al. (1979) [9], A. Brugen (1981) [10], A. Gero (1983) [11], and other outstanding scientists. Thanks to their work the classical theory was significantly extended and many phenomena of ligand-receptor interactions were explained. However, the causes of variability in the slope "dose (concentration)-effect" dependence remained unsolved, like other phenomena found out in pharmacological in vivo and ex vivo experiments, including bimodal and polymodal "dose (concentration)-effect" dependence, obtained in vivo controversial of in vitro linear nature of the ligand-receptor binding. It should be noted that the current trend in receptorology development to avoid simplistic representations of BACs effects and the work of biological systems was accepted [12-14], so recently in the scientific terminology the concept of pleiotropic activity was introduced, for example, in respect of G-proteins action [15] and the segregate drugs effects $[16,17]$. Regarding the latter, it is worth noting that the authors use the term "pleiotropy" in a different context, meaning a property that can be rather defined as "multiaffinity" (or "polytropism"). The differences between these tow aspects of the BACs action are discussed below. A group of researchers under the direction of R. Tallarida, a well-known expert in the field of combinatorial pharmacology, approached to the nature of pleiotropic action in such interpretation. They completed and in 2011 published the findings of research concerning the synergistic mechanisms of tapendalol analgesic effect [18].

Pleiotropic activity and multiaffinity of BACs and medicines. Based on the understanding of pleiotropy, as the ability of the BACs and drugs to cause certain biological (pharmacological) effect due to the realization of several activity mechanisms at the range of effective concentrations, it is clear, that the molecular nature of the pleiotropic activity is multifarious and entirely dependent on the chemical structure and the related physical and chemical properties of the compound. As for the antioxidant effect, studied by the author, pleiotropic activity combines antiradical properties and pharmacodynamic effects in the cellular structures and effectors, resulting in some way, in the decrease of the ROS quantitative yield, involving Nox2-dependent generation. It should be noted, that the direct antiradical effect, in its turn, can be regarded as pleiotropic, since the vast majority of antioxidants can interact with several types of free radicals. Discussion of pleiotropic antioxidant effects is beyond the scope of this article, as it was carried out earlier [19].

Pharmacodynamic pleiotropy will primarily be considered in the relation to receptor agonists as the most widely used medicines. It is composed of relative recaptor selectivity of these compounds on the one hand, and the "plasticity" of receptors themselves, on the other hand. However, pleiotropic nature is not limited to this. As it is vividly seen from the research of the last half-decade, many receptors, and possibly all, inherent relative tropism to the different intracellular signaling pathways with the potential to alternative signaling through GP-switch [20]. The example of pleiotropic action due to this mechanism may be isoprenaline effect, as the agonist of $\beta$ adrenergic receptors $(\beta$-ARs). Its stimulation, according to modern ideas, should lead to Gs-mediated increase in the intracellular concentration of cyclic adenosine monophosphate (cAMP) and the strong inhibitory effect for Nox2-dependent ROS production [21]. However, in fact, the effect is below the expected yield. Magocsi M. et al. research [20] allowed to explain it by demonstrating the fact, which the tropism of the $\beta$-ARs to Gs-protein is also relative and in a variety of conditions $\beta$-ARs interacts with Gi-protein activating phosphoinositol-3-phosphate (PIP3) cascade, alternative cAMP, and is able to neutralize the effects of its activation [21]. The probability of Gs/Gi-switching increases with the increment in the number of activated $\beta$-ARs [20]. This is consistent in the present study with the observed pattern of eliminated pleiotropic action of isoprenaline (see below) and can, at least partially, explain its nature. Currently, the causes and mechanisms of GP-switching are poorly understood, but they certainly make a significant contribution to the pleiotropic biological response and may explain, in addition, some of the contradictions between the resulting pharmacological efficacy of the number of receptor agonists and receptor theory postulates.

Biological significance also has polytropism (polytropic action), another property of BACs and medicines, which should be understood as the ability to interact with several relatively autonomous molecular targets and cause several independent pharmacological effects, each being pleiotropic. It is important to emphasize that polytropism is a property of the BACs, whereas the concept of pleiotropy can rather be referred to the characteristics of its effects. Polytropism can be the basis of pleiotropic activity, when multiple targets of BACs responsible for development of the same effect. A typical example of polytropic compounds are 7-TMS receptors non-selective agonists, such as dopamine. Dopamine has polytropism because it can stimulate different receptors (dopamine and adrenergic) and cause independent, often alternative, effects. So, in one range of concentrations (doses) it stimulates dopamine receptors in the kidney vessels, reducing vascular tone and increasing renal blood flow, 
while in the other, it stimulates alpha-adrenergic recaptors of the same vessels, thus leading to the alternative effect. Moreover, each of the effects may be pleiotropic. For example, above mentioned vasodilation effect of dopamine is ensured not only by "fast" signals from the dopamine receptors in the appropriate intracellular effectors, but also by the delayed effects caused by arrestin activation [22], as well as by the modification of ROS-dependent intercellular signaling.

Another typical example is nifedipine. In contrast to isoprenaline, nifedipine realises the amplifying pleiotropic pattern (see below) for Nox2-dependent ROS generation in the macrophages, which can be explained by a dose-dependent blocking $\mathrm{Ca}^{2+}$ channels, on the one hand, and the inhibition of the enzyme protein kinase $\mathrm{C}$ (PKC), on the other hand [23], given, that both effects inhibit the activity of Nox2. Verapamil, in contrast to nifedipine did not inhibit PKC and for Nox2-dependent ROS generation realizes a different pattern-parallel pleiotropy. Thus, nifedipine is a polytropic compound, pleiotropy of which appears only in case of a particular activity, i.e, inhibiting effect on Nox2-dependent ROS generation in macrophages, which is provided by the exposure of the two responsible for this process molecular targets. In a common case, the necessary condition to form pleiotropy on the basis of polytropism is the accessibility of several molecular targets for the effector and their simultaneous participation in the development of the studied effect. Pleiotropy may be provided by the interactions of specific and non-specific mechanisms of BACs. Modern research in the field of high energy chemistry suggests that free radical fragmentation of the polar lipids is not only important for biological membrane disruption, but it also ensures the release of BACs acting as second messengers in the cells [24]. In this regard, presence of the own prooxidant or antioxidant activity of a BACs along with specific activity determines its resulting biological effect. Propranolol may be taken as an example of such compounds. It is proved that the cardioprotective effect of propranolol is provided by its blockage on the heart $\beta$-ARs, on the one hand, and powerful antiradical activity for carbon-centered radicals, on the other hand [25]. In this aspect, pleiotropy, in essence, is a manifestation of the internal interactions, initiated by the mechanisms of intra- and intercellular signaling and communication. It should be emphasized that the pleiotropic pattern is determined by the molecular mechanisms of the BACs, but their study and analysis is not sufficient for the effective practical application of them. Of primary interest are pleiotropic patterns themselves, since they offer the possibility of meaningful control of the effectory system (effect). In this respect, the properties of the models chosen to assess the effect of BACs and medicines from the point of pleiotropy are of great importance. Returning to the research, the findings of which form the basis of this work, it should be noted, that it is the choice of the relevant model that allowed us to define and study the patterns of tested bioactive compounds and antioxidants pleiotropic actions for Nox2dependent ROS generation in macrophages.

\section{MATERIALS AND METHODS}

\subsection{Nox2-Dependent ROS Generation Research}

The model to study of the Nox2-dependent ROS generation were resident peritoneal rat macrophages. This research is based on the testing findings of 140 individual compounds and 80 combinations of the BACs and pharmacological agents in a wide range of concentrations $\left(10^{-3}-10^{-8} \mathrm{M}\right)$. The influence of the test compounds and their combinations on total and Nox2-dependent ROS generation in macrophages was studied separately by luminol-dependent chemiluminescence (CL) in experiments in vitro on the LKB Wallac-1251 Luminometer (Sweden). As an inducer of the respiratory burst, opsonized zymosan (OZ) was used, the testing time of pre-incubation compounds with macrophages was 10 minutes, while the time of CL registration was 40 minutes. The total (cumulative) number of ROS generated by cells was assessed by the area under the CL curve (AUC CL), mV/106 cells. The indicator of the Nox2-dependent pool contribution in total cellular ROS production was described as DAUC $\mathrm{CL} \mathrm{mV} / 106$ cells and calculated as the difference between AUC CL and the part which is due to the ROS generation is beyond Nox 2 activation (provided by other sources of ROS). The CL sample indicators, containing the compounds under study, were expressed in the percentage to the control samples. The effect of each modulator and combination was reproduced from 3 to 8 times.

\subsection{Statistical Processing and Analysis}

Statistical processing of the primary outcome in the series was performed using paired t-test, interseries comparisons were made by the Student's t-test criterion. In all cases, the difference was considered significant if the probability of error was less than $5 \%(p<0.05)$. Antioxidant activity of the compounds was assessed by the degree of CL suppression, calculating the effective inhibitory concentrations (IC16 - IC84) by the linear regression using PAP "Statistica 6.1" and the "Microsoft Office Exel 2010" algorithm to analyze the interaction of component combinations by the Chou-Talalay combination index (CI) method [2]. In general, the equation of the combination index is expressed as follows (1):

where $(D)_{j}$-agent dose (concentration) producing the effect of a certain strength in case of combined use; $\left(D_{x}\right)_{j}$ 


$$
C I=\sum_{j=1}^{n} \frac{(D)_{j}}{\left(D_{x}\right)_{j}}
$$

-agent dose (concentration), producing a similar effect in case of the individual application; $n-$ the number of components in a combination.

Assessment of synergy and antagonism according to Chou-Talalay is as follows: $\mathrm{CI}<0.1-$ very strong synergism; 0.1 - 0.3 - strong synergism; 0.3 - 0.7 - synergism; 0.7 - 0.85-moderate synergism; $0.85-0.90$-weak synergism; 0.90 - 1.10 - additive effect; 1.10 - 1.20-weak antagonism; 1.20 - 1.45-moderate antagonism, 1.45 3.3-antagonism; $3.3-10.0$ - strong antagonism, $>10$ very strong antagonism.

\subsection{Pleiotropy Analysis}

The most important feature of the model, chosen to assess pleiotropy, is a differentiated analysis of the test compound activity relative to the total process, and to the part of the process provided by the activity of the particularly effector's. So, analysis of the pleiotropic patterns in this study was made with a simple, yet highly informative model. Its essence is to determine the ratio of the test compound (or combination of compounds) effect in relation to Nox2-dependent ROS generation activity referring to the total process of cell ROS generation. This ratio has been called the coefficient of relative activity (pleiotropic activity, $\mathrm{K}_{\mathrm{x}}$ ). On the basis of the calculation data in the range of effect from $E_{16}$ to $E_{84}$ for compounds inhibiting Nox2-dependent ROS generation in macrophages the pleiotropic pattern $\left(\mathrm{K}_{16-84}\right)$ was assessed. The coefficients $\mathrm{K}_{16-84}$ were calculated according to the formula (2):

$$
K_{x}=\frac{I C_{x A U C}}{I C_{x D A U C}}
$$

$\mathrm{IC}_{\mathrm{x}}$ was calculated by the equation [2] (3):

$$
I C_{x}=I C_{50}\left[f_{a} /\left(1-f_{a}\right)\right]^{1 / m}
$$

where $K_{x}$ is the pleiotropic activity coefficient at the $X$ level of the effect; $I C_{X A U C}$ - inhibitory concentration of the compound in relation to the total ROS generation at the $X$ level of the effect; $I C_{x D A U C}$-inhibitory concentration of compound for Nox2-dependent ROS generation at the same effect level; $f_{a}$-affected fraction from the maximum effect; $1-f_{a}\left(f_{u}\right)$-unaffected fraction from the maximum effect; $m$ - the slope coefficient of the "concentration-effect" dependence.

It is seen from the above equation that the pleiotropic pattern demonstrates a Nox 2 contribution to the formation of the total pool ROS generated by cell at different concentrations of the BACs. Thus, if the Nox 2 contri- bution growth increased symbatically to the modulator concentration, a pattern, called sequential amplified (synergistic) pleiotropy (SAP-pattern), was shaped. This pattern corresponded to the exponential increase of $\mathrm{K}_{16-84}$ with the increase of the BACs efficiency, which is clearly seen in Figure 2(a). The results are presented in semilogarithmic coordinates $\left(\log K_{x}-E_{x}\right)$, this pattern corresponds to the positive values of the slope of the pleiotropic pattern to the $\mathrm{x}$-axis, on which biological effectiveness of the active compound ( $\operatorname{tg}>0.1$ ) (Figure 2(b)).

On the contrary, if Nox 2 contribution to the ROS cell generation decreased with the increase of the modulator concentration, the sequential eliminated (antagonistic) pleiotropic pattern (SEP-pattern) was formed. Exponential $\mathrm{K}_{16-84}$ decrease with the increase of the BACs efficiency was consistent with this pattern. In the process of data processing in the $\left(\log \mathrm{K}_{\mathrm{x}}-\mathrm{E}_{\mathrm{x}}\right)$ coordinates, the

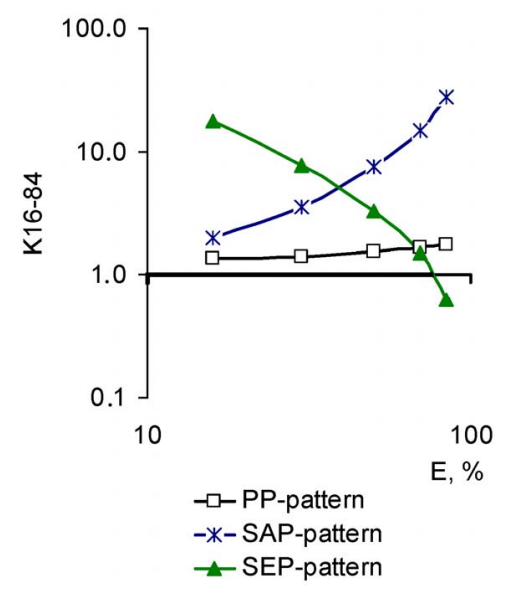

(a)

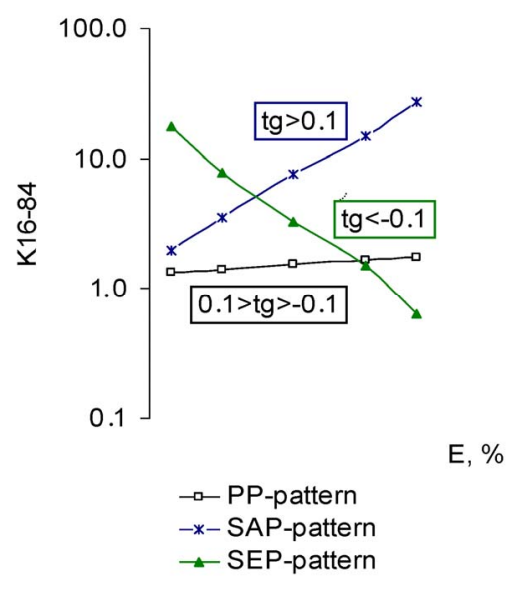

(b)

Figure 2. Basic pleiotropic patterns of BACs and drugs for Nox2-dependent ROS generation by macrophages in FcRinduced phagocytosis. (a) Logarithmic coordinates $\left(\log \mathrm{K}_{\mathrm{x}}-\log \mathrm{E}_{\mathrm{x}}\right.$ ); (b) Semilogarithmic coordinates $\left(\log K_{x}-E_{x}\right)$. 
slope of the pleiotropic pattern in the range $\mathrm{K}_{16-84}$ to the $\mathrm{x}$-axis becomes negative $(\operatorname{tg}<-0.1)$.

In that case, the contribution of Nox 2 in the ROS cellular generation remained unchanged as the increase in the concentration of the BACs (a constant fraction at each level of the effect) the parallel pleiotropic pattern (PPpattern) was formed that matched the $\mathrm{K}_{16-84}$ constant in the effective dose range. In case the results were presented in semilogarithmic coordinates, this pattern is determined by the value of the $\operatorname{tg}$ of +0.1 to $-0.1(0.1>\operatorname{tg}>$ -0.1 ). In general, the pleiotropic activity is a fraction of the BACs action for the target and cumulative effect, including the target effect. Thus, pleiotropic activity is not the characteristic of the effectors (BACs), but their effect in the particular biological circumstances (test-systems).

Another essential property of the model is the effect assessment of by the parameters allowing take into account a set of the tested compound activity mechanisms. This study considered a quantitative yield of the final products, determined by the activity of Nox 2 . The tested compound could in any way modify the ROS output, ranging from the direct anti-radical activity and finishing with the enzyme-generator assembly violation, while creating a specific pattern that doesn't characterize mechanisms as such, but the nature of their interaction on Nox2-dependent ROS generation. In the context of the diagnostics (analysis) it should be emphasized that we speak about pleiotropic activity in respect of a certain effect, in this case, inhibition of Nox2-dependent ROS generation. In addition, pleiotropic patterns are estimated in each effect separately for polytropic compounds.

\section{RESULTS AND DISCUSSION}

\subsection{Pleiotropic Patterns}

The test results of the biologically active set of compounds with different types of activity, inhibiting Nox2dependent ROS generation in macrophages, have revealed three main patterns of pleiotropic action. The characteristics of these patterns are as follows. The first-PPpattern, differs by the constancy of the BACs pleiotropic activity in the whole range of effective concentrations. It suggests a rapid simultaneous operation of the BACs mechanisms of activity and mechanisms of biosystems reactivity keeping this consistency throughout the effective range. This pattern may also be formed by the compounds the pleiotropy of which is not possible to be identified in the proposed terms and because of this, they appear to be "monotropic". In the present model the condition under which a PP-pattern is formed, is the constant activity relating to the Nox2-dependent ROS generation in any concentrations of the biologically active compounds, the slope angles of the "concentration effect" for total and Nox2-dependent ROS generation are identical (Figure 3). In the present research, this pattern was exhibited by $\beta$-adrenergic antagonists (propranolol, sotalol, oxprenolol, metoprolol) and serotonin (ondansetron) receptors, sodium channel blockers (procaine, bupivacaine), verapamil, ATP, serotonin, and the amino acid L-arginine and antioxidants with high activity for carbon-centered radicals (compound C16 - 5-methoxy-1,3benzoxatiol-2-thione). In its turn, it is possible to identify the subvariants of the PP-pattern based on the tropism mechanisms of its form to the target effector (in this case Nox2) and alternative effectors, together making up a complex response, such as ROS cellular production (Figure 3):

- Pattern of parallel symbatic pleiotropy (PPs-pattern) with $\mathrm{K}_{16-84}>1.0$ demonstrates a high tropism for the target effector;

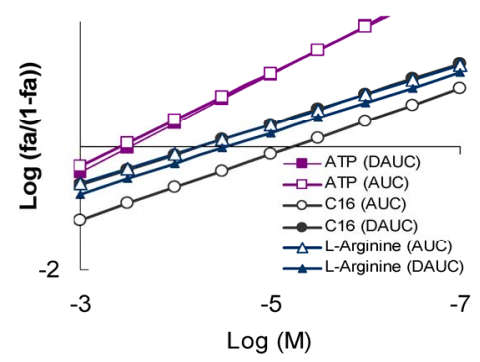

(a)

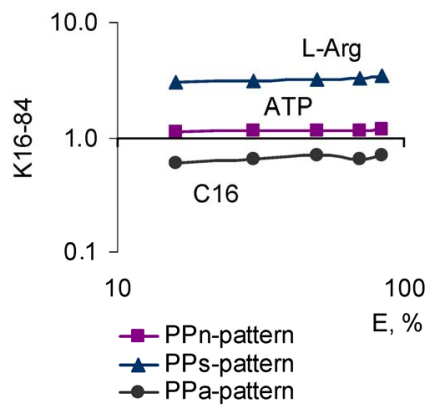

(b)

Figure 3. The character of the "concentration-effect" dependence for ATP, L-arginine and compound C16 (top) and corresponding subvariants of the parallel pleiotropic pattern (bottom). (a) "Concentration-effect" dependence for compounds that show different pattern subvariants of parallel pleiotropy (Hill's coordinates). AUC-activity against the total ROS cell generation, DAUC-activity against its Nox2-dependent part; (b) Subvariants of the parallel pleiotropic pattern on the example of ATP (parallel neutral), L-arginine (parallel symbatic), C16 compounds (parallel antibatic). Logarithmic coordinates. 
- Pattern of parallel antibatic pleiotropy (PPa-pattern) with $\mathrm{K}_{16-84}<1.0$ demonstrates a high tropism to alternative effectors;

- Pattern of parallel neutral pleiotropy (PPn-pattern) with $\mathrm{K}_{16-84} \approx 1.0$ demonstrates comparable tropism for the target and alternative effectors.

The second is SEP-pattern features intibatic efficiency increase, exponential decrease of BAC pleiotropic activity in case when its concentration increases.

This pattern is formed by a sequential, dose-dependent switching on of the BACs molecular mechanisms and mechanisms of biological system reactivity, interacting with each other on the basis of antagonism. In the conditions of the used model it is formed in sequential, dependent on the concentration inclusion of various antagonistic or competing mechanisms of a Nox 2 modification dependent process, determining the exponential fall in activity with respect to this component of the total ROS cellular generation. The angle of the "concentration-effect" curve slope for total ROS generation exceeds that of the Nox2-dependent process (Figure 4).

For each BAC the mechanism of the pattern formation is different, however, all of them lose their activity for Nox2-dependent ROS generation with the increase of their concentration. Only a few compounds of different pharmacodynamic profile demonstrated this type of pleiotropy, including isoprenaline, AMP, taurine, colchicine.

The third-SAP-pattern is characterized by symbatic efficiency exponential increase of the BAC pleiotropic activity with the growth of its concentration. The condition for this pattern formation is the sequential, dosedependent incorporation of the molecular mechanisms of BACs and of biological systems reactivity mechanisms, interacting with each other synergistically. In the present study the condition for the formation of this pattern is sequential, concentration-dependent incorporation of synergistic effects for Nox2-dependent ROS generation defining exponential growth of activity to the components of total ROS cellular generation. In contrast to the previous pattern, the "concentration-effect" slope angle for the total ROS generation is less than for Nox2-dependent one (Figure 5). In the present study many receptor agonists demonstrated that type of pleiotropic pattern, including antioxidants, with high antiradical selectivity for oxygen-centered radicals, or combining this activity with pharmacodynamic antioxidant action.

\subsection{Correlation between the Pleiotropic Pattern and the Slope of the "Concentration- Effect” Dependence}

Comparative analysis of the "concentration-effect" dependence slope in the ROS macrophages generation inhibitors showed that in assessing the effects of the overall process, the greatest slope angle (maximum values tg)

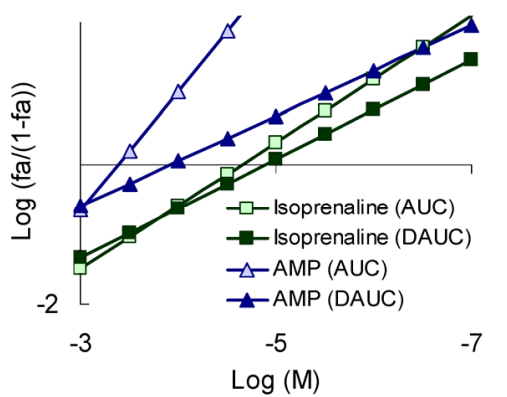

(a)

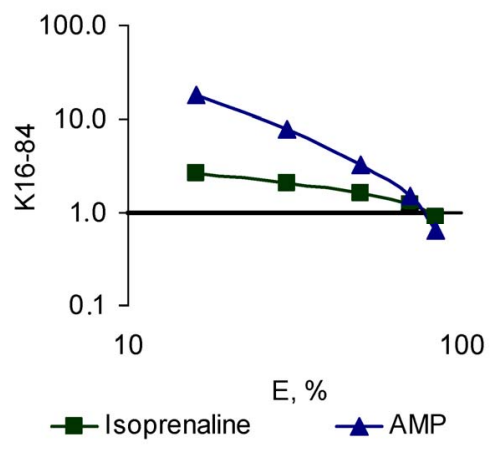

(b)

Figure 4. The character of the "concentration-effect" dependence for isoprenaline and AMP (top) and the corresponding pattern of the sequential eliminated (antagonistic) pleiotropy (bottom). (a) "Concentration-effect" dependence for isoprenaline and AMP (Hill's coordinates). AUC-activity for total ROS generation, DAUC-activity for its Nox2-dependent part; (b) General view of a sequential eliminating (antagonistic) pleiotropic pattern on the example of isoprenaline and AMP. Logarithmic coordinates.

demonstrated the compounds with a SEP-pattern for Nox2-dependent generation (isoprenaline, AMP), while the smallest angle was demonstrated by the compounds with a SAP-pattern (lidocaine, nifedipine); compounds with PP-pattern had intermediate values of the slope angle (Figure 6).

When we assessed the effect of Nox2-dependent ROS generation the reversed picture was obtained. In this system, the largest angles showed the compounds with a SAP-pattern (lidocaine, nifedipine), and the lowest angle demonstrated the compounds with the SEP-pattern (isoprenaline, AMP). Modulators realizing the PP-pattern still had intermediate values of the slope angle (Figure 7).

In general, the "dose (concentration)-effect" slope angle depends on the test systems of the studied modulators. If the experimental conditions allow to assess the effect of the modulator with respect to the total process consisting of $\mathrm{n}$ certain individual (target) processes, the slope 


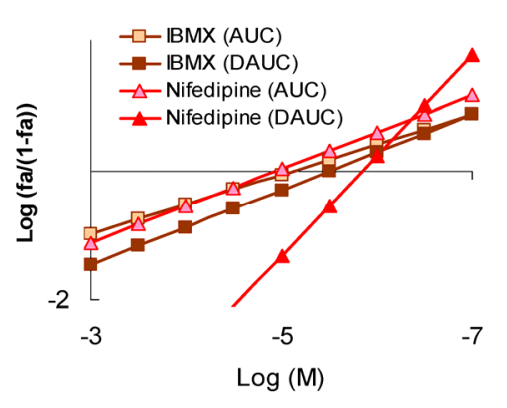

(a)

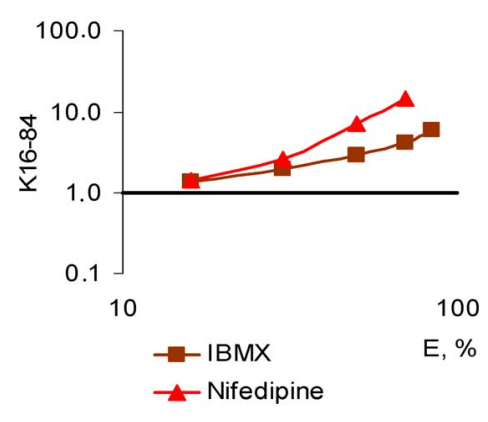

(b)

Figure 5. The character of the "concentration-effect" for isobutyl-methylxanthine (IBMX) and nifedipine (top) and the corresponding pattern of the sequential symbatic amplifying (synergistic) pleiotropy (bottom). (a) "Concentration-effect" dependence for IBMX and nifedipine (Hill's coordinates). AUC-activity for total ROS generation by cells, DAUC-the activity for its Nox2-dependent part; General view of a sequential amplifying (synergistic) pleiotropy pattern on the example of IBMX and nifedipine. Logarithmic coordinates.

angles of "dose (concentration)-effect" dependence will tend to be less in the compounds demonstrating a SAPpattern and bigger in a SEP-pattern. It is logical to assume that the larger $n$, the smaller the "dose (concentration)-effect" slope angle for BACs. For the individual (target) situation it is quite the opposite process - the "dose (concentration)-effect" slope angle is bigger in case of the SAP-pattern and smaller in those with antagonistic pleiotropy (SEP-pattern). Compounds that show a PP-pattern occupy the intermediate place in both test systems.

To a great extend the above ideas are in tune with the ideas of Hill who offered to assess the potential of interaction between the receptors according to the tangent slope angle similar lines obtained for the receptor agonists and introduced in receptology the concept of noncooperative receptor interaction $(\operatorname{tg}=1)$, positive $(\operatorname{tg}>1)$ and negative $(\operatorname{tg}<1)$ cooperativity. However, at that time
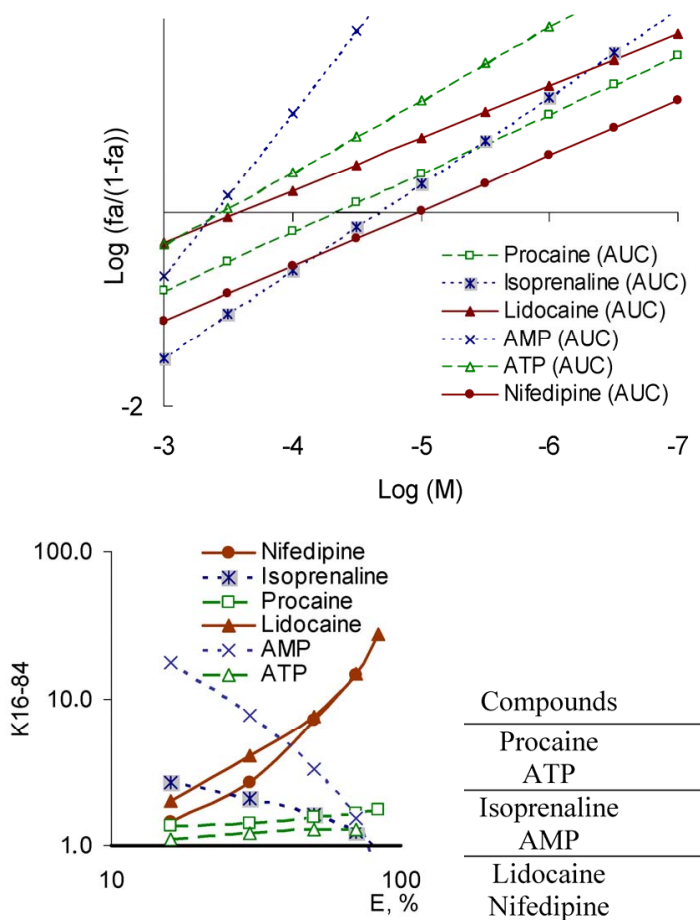

\begin{tabular}{c|c} 
Compounds & $\operatorname{tg} \alpha$ \\
\hline Procaine & 0.48 \\
ATP & 0.58 \\
\hline Isoprenaline & 0.70 \\
AMP & 1.37 \\
\hline Lidocaine & 0.40 \\
Nifedipine & 0.44
\end{tabular}

Figure 6. "Concentration-effect" dependence in Hill's coordinates on the model of total ROS generation (top) and the corresponding values of tg angle (bottom right) for a number of modulators realizing different patterns of pleiotropy for its Nox2-dependent part (bottom left).
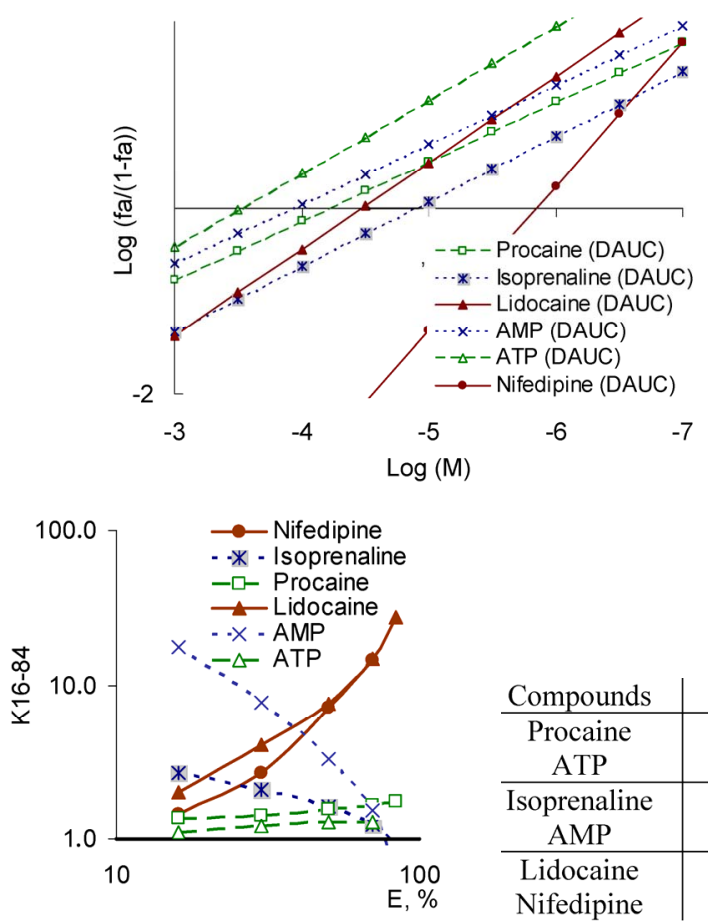

\begin{tabular}{c|c} 
Compounds & $\operatorname{tg} \alpha$ \\
\hline Procaine & 0.47 \\
ATP & 0.58 \\
\hline Isoprenaline & 0.47 \\
AMP & 0.44 \\
\hline Lidocaine & 0.64 \\
Nifedipine & 1.11
\end{tabular}

Figure 7. "Concentration - effect" dependence in the Hill coordinates on the model of Nox2-dependent ROS generation (top) and the corresponding values of tg angle (bottom right) for a number of modulators that realize different patterns of pleiotropy (bottom left). 
the development of the receptor theory and the interpretation of research findings of receptor agonists based on Hill postulates were limited by rather simplistic notions about their effects in vivo and they did not take into account a wide range of biochemical mechanisms, realized apart from receptor bindings. Due to the same reason receptor antagonists and compounds of unknown type of activity were also not taken into account. In the light of the theory of pleiotropic action, the interaction of receptors can only be considered as one of the mechanisms of pleiotropy.

\subsection{Justification of Pleiotropic Patterns Using the Chou-Talalay Theory of the Combination Index}

The above concept of pleiotropic patterns could be classified as a hypothesis, if there were not the evidences obtained in the experiments concerning the combinations of BACs. These studies were based on the practical application of the Chou-Talalay theory of the combination index, developed by the authors more than 30 years ago and thoroughly tested in the world experimental practice [2]. While studying of nearly a hundred different combinations of BACs with the assessment of pleiotropic patterns with respect to their inhibitory effect on Nox2-associated ROS generation it became possible to establish the following. The greater the number of cross-mechanisms of action provides the combination effect, the more patterns of combinations differ from patterns of prototypes. More than that, it is not possible to predict the combination pattern by the prototypes patterns, as well as it is not possible to predict the outcome of their pharmacodynamic interactions [1,2]. However, based on the pattern combination matching, and the character of their components interaction, we can prove the nature of pleiotropy individual patterns, which has so far been considered only in the framework of the hypothesis. Without taking into account the absolute values of the combination index $(\mathrm{CI})$ and related interpretations of the interaction findings and consider only $\mathrm{CI}$ dynamics, as a basic feature in the process of the growth effect (and concentration) of the active compound, the observed pattern should look in the following way. If there components demonstrate dose-dependent synergistic interaction regarding the Nox2-dependent ROS generation, the combination should form a SAP-pattern, and in case of dosedependent antagonistic interaction - a SEP-pattern should form.

This thesis is fully consistent with the experimental results. A combination, the component synergy of which increases with the increase of concentrations, regardless of the prototypes patterns, realized two pleiotropic patterns-amplified (SAP-pattern) or parallel (PP-pattern). In contrast, the combinations in which with the increase of concentration antagonism of ingredients has grown (even the initial synergies at $\mathrm{E}_{16-30}$ ) have formed the SEPpattern. The examples of combinations of the first and second type are shown in Figure 8.

\subsection{Place of the Theory of Pleiotropic Action in Modern Pharmacology}

Analysis of the Experimental Pharmacology historical experience and the current situation show that the weak point of modern scientific research is extremely high (analytical) specialization of individual scientific fields and almost complete absence of synthetic approaches mutually integrating their knowledge and thus opening the prospects for effective practical application of fundamental scientific advances. Relating to the dose regularities of drug use, the main mistake is to identify the effect, caused by a specific receptor binding with the resulting effect of the BACs and medicines, composed of the effector activity set of mechanisms and the reactivity mechanisms of the biological system itself. The novel theory of pleiotropic action resolves this conflict, focusing on the nature of the "dose (concentration)-effect" dependency from the point of view that the slope angle is an integral indicator of the BACs and medicines interaction with biosystems (bioeffectors) on the basis of their functional plasticity. This explains not only the difference between the slope angles for different medicines in the same test system, but also the differences of angles for the same medicine in different test systems. Analysis of the medicine effects from the point of view of this theory allows to explain both the pleiotropy as the absolute difference in the "dose-effect" slope dependences, set in a specific test system, and the relative differences in the slope, potency and efficacy found when the effects relating to the total process, and the its target components were compared. The novel theory also explains bimodal (polymodal) "dose-effect" dependences in pharmacological experiments, as opposed to the linear dependence obtained in biochemical research in vitro. The theory of pleiotropic action is complementary to the receptor theory and the theory of Chou-Talalay combination index. Together with them it enhances understanding the essence of the biological effects of BACs and medicines and their combinations, and allows explore the most effective ones, with a high tropism to target components of complex biological processes, the search of approaches to modulation of which activity is the essence of research in a special pharmacology.

\section{CONCLUSION}

The theory of pleiotropic action of biologically active compounds and medicines explains unsolved until now 

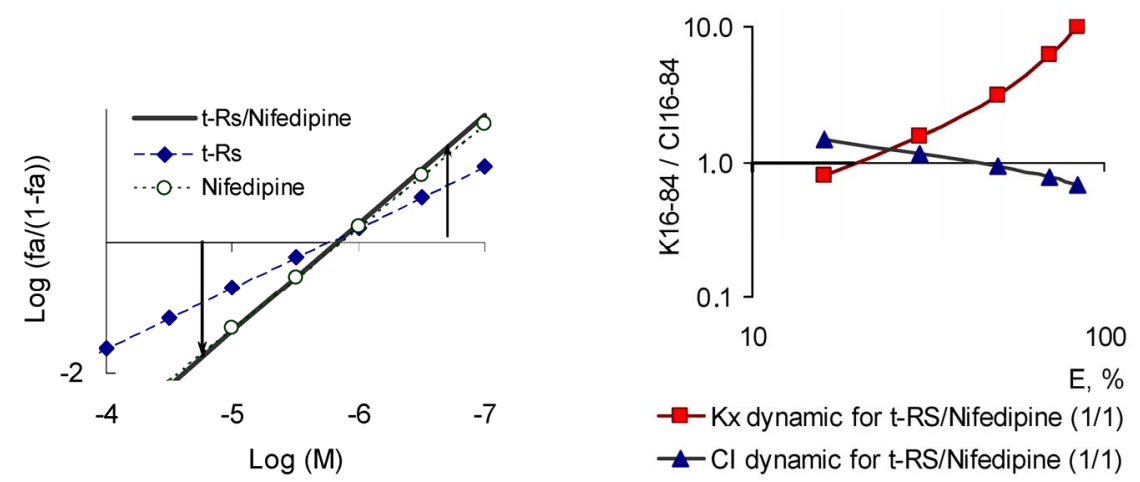

(a)
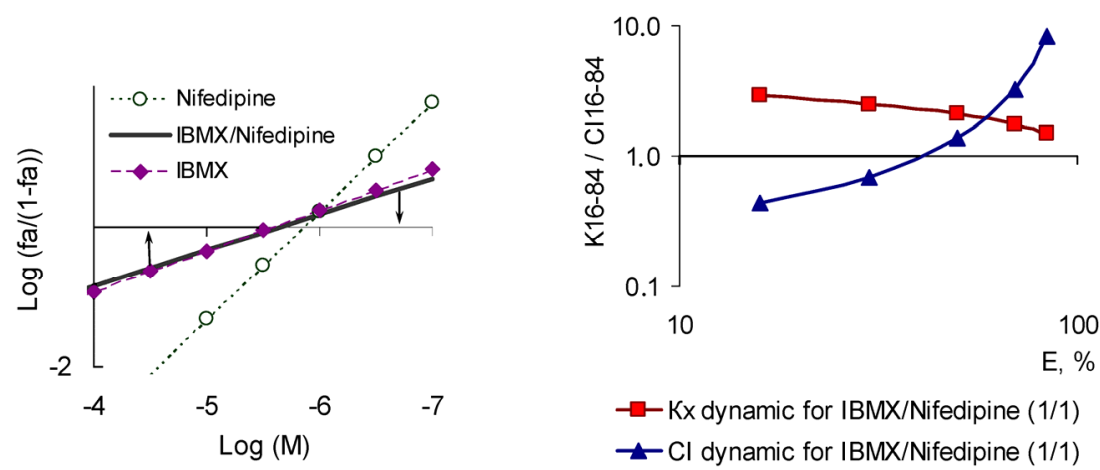

(b)

Figure 8. Characteristics of "concentration-effect" dependency (for Nox2-dependent ROS generation), pleiotropic patterns ( $\mathrm{K}_{16-84}$ dynamic) and the corresponding patterns of the combination index $\left(\mathrm{CI}_{16-84}\right.$ dynamic), characteristic to the combinations of BACs, differed by the potential of pharmacodynamic interactions. (a) Combination of nifedipine and trans-resveratrol (in the molar ratio 1/1), the components of which demonstrate a dose-dependent increase in a pharmacodynamic synergy $\left(\mathrm{CI}_{16-84}\right.$ dynamic), corresponds the SAP-pattern (right), logarithmic coordinates; the slope angle of the curve "concentration-effect" dependence for the combination is increased compared with the components (left, Hill coordinates); (b) IBMX and nifedipine combinations (in the molar ratio $1 / 1)$, the components of which demonstrate a dose-dependent increase in pharmacodynamic antagonism $\left(\mathrm{CI}_{16-84}\right.$ dynamic), corresponds the SEP-pattern (right, logarithmic coordinates); the slope angle of the curve "concentration-effect" dependence is decreased related to the combination of the individual components (left, Hill coordinates).

universal aspects of their activity in the conditions relevant in vivo, and in this respect it is of fundamental interest. However, it has applications in experimental pharmacology, as it allows defining the choice of the individual compounds and combinations, modulating the trust effect selectively and efficiently. This knowledge opens up new approaches to medicines discovery and evaluation, their rational dosing and combining.

\section{ACKNOWLEDGEMENTS}

The author expresses his deep gratitude to Professor Boris V. Dubovik, Doctor of Medical Sciences, Laureate of the State Prize of the USSR, who did much for the establishment and development of the research, the findings of which form the basis of this work, and for the fruitful discussion and advices, given in the process of this article getting ready for publication.

\section{REFERENCES}

[1] Tallarida, R.J. (2000) Drug synergism and dose-effect data analysis. Chapman and Hall/CRC, Roca Raton. doi:10.1201/9781420036107

[2] Chou, T.-Ch. (2006) Theoretical basis, experimental design, and computerized simulation of synergism and antagonism in drug combination studies. Pharmacological reviews, 58, 621-681. doi:10.1124/pr.58.3.10

[3] Sergeev, P.V., Szymanowski, N.L. and Petrov, V.I. (1999) Receptors of physiologically active substances. 2nd Edition, "Seven Winds" Publishing House, Volgograd.

[4] Stephenson, R.P. (1956) A modification of receptor theory. British Journal of Pharmacology, 11, 379-393. 
[5] Paton, W.D. (1961) A theory of drug action based on rate of drug-receptor combination. Proceedings of the Royal Society B: Biological Sciences, 154, 21-69. doi:10.1098/rspb.1961.0020

[6] Karlin, A. (1967) On the application of "a plausible model" of allosteric proteins to the receptor for acetylcholine. Journal of Theoretical Biology, 16, 306-320. doi:10.1016/0022-5193(67)90011-2

[7] Colquhoun, D. (1973) The relation between classical and cooperative models for drug action. In: Rang, H.P., Ed., Drug Receptors, Macmillan Press, London, 149-182.

[8] Colquhoun, D. (1998) Binding, gating, affinity and efficacy. The interpretation of structure-activity relationships for agonists and of the effects of mutating receptors. British Journal of Pharmacology, 125, 923-948. doi:10.1038/sj.bjp.0702164

[9] De Lean, A., Stadel, J.M. and Lefkowitz, R.J. (1980) A ternary complex model explains the agonist-specific binding properties of the adenylate cyclase-coupled beta-adrenergic receptor. The Journal of Biological Chemistry, 255, 7108-7117.

[10] Brugen, A. (1981) Conformational changes and drug action. FedProc, 40, 2723-2728.

[11] Gero, A. (1983) Desensitization, two-state receptors and pharmacological parameters. Journal of Theoretical Biology, 103, 137-161. doi:10.1016/0022-5193(83)90204-7

[12] Urban, J.D, Clarke, W.P., Von Zastrow, M., Nichols, D.E., Kobilka, B., Weinstein, H., et al. (2007) Functional selectivity and classical concepts of quantitative pharmacology. The Journal of Pharmacology and Experimental Therapeutics, 320, 1-13. doi:10.1124/jpet.106.104463

[13] Black, J.W., Leff, P., Shankley, N.P. and Wood, J. (2010) An operational model of pharmacological agonism: The effect of $\mathrm{E} /[\mathrm{A}]$ curve shape on agonist dissociation constant estimation. British Journal of Pharmacology, 160, S54-S64. doi:10.1111/j.1476-5381.2010.00855.x

[14] Kenakin, T. (2004) Principles: Receptor theory in pharmacology. Trends in Pharmacological Sciences, 25, 186192. doi:10.1016/i.tips.2004.02.012

[15] Strange, P.G. (2008) Agonist binding, agonist affinity and agonist efficacy at $\mathrm{G}$ protein-coupled receptors. British Journal of Pharmacology, 153, 1353-1363. doi:10.1038/sj.bjp.0707672

[16] Lambeth, J.D. (2007) Nox enzymes, ROS, and chronic disease: An example of antagonistic pleiotropy. Free Radical Biology \& Medicine, 43, 332-347. doi:10.1016/j.freeradbiomed.2007.03.027

[17] Ito, M.K., Talbert, R.L. and Tsimikas, S. (2006) Statinassociated pleiotropy: Possible beneficial effects beyond cholesterol reduction. Pharmacotherapy, 26, 85S-97S. doi:10.1592/phco.26.7part2.85S

[18] Schroder, W., Tzschentke, T.M., Terlinden, R., De Vry, J., Jahnel, U., Christoph, T. and Tallarida, R.J. (2011) Synergistic interaction between the two mechanisms of action of tapentadol in analgesia. The Journal of Pharmacology and Experimental Therapeutics, 337, 312-320. doi:10.1124/jpet.110.175042

[19] Bizunok, N.A. (2013) Structural determinants of antioxidative activity of the phenols, diphenols and polyphenols at reactive oxygen species generated by macrophages in a different microenvironment circumstances. Military $\mathrm{Me}$ dicine, 1, 84-94. http://www.bsmu.by/files/vm/1-2013/24.pdf

[20] Magocsi, M., Vizi, E.S., Selmeczy, Z., Brózik A. and Szelenyi, J. (2007) Multiple G-protein-coupling specificity of $\beta$-adrenoreceptor in macrophages. Immunology, 122, 503 513. doi:10.1111/j.1365-2567.2007.02658.x

[21] García-García, E. and Rosales, C. (2002) Signal transduction during Fc receptor-mediated phagocytosis. Journal of Leukocyte Biology, 72, 1092-1108.

[22] Luttrell, L.M. and Lefkowitz, R.J. (2002) The role of beta-arrestins in the termination and transduction of G-protein-coupled receptor signals. Journal of Cell Science, 115, 455-465.

[23] Allanore, Y., Borderie, D., Perianin, A., Lemaréchal, H., Ekindjian, O.G. and Kahan, A. (2005) Nifedipine protects against overproduction of superoxide anion by monocytes from patients with systemic sclerosis. Arthritis Research \& Therapy, 7, R93-R100. doi:10.1186/ar1457

[24] Yurkova, I.L. (2010) Free radical fragmentation in the polar part of the lipids: A new path of destruction and the formation of biologically active compounds. Dissertation for the Degree of Doctor of Chemical Sciences, Belarusian State University, Minsk.

[25] Tong Mac, I., Arroyo, M.C. and Weglicki, W.B. (1989) Inhibition of sarcolemmal carbon-centered free radical formation by propranolol. Circulation Research, 65, 11511156. doi:10.1186/ar1718 